\title{
Equifinal Throwing Movements - Computational Approach, Mechanical Implementation, and Learning Intervention
}

\author{
Mathias Reiser ${ }^{*} \quad$ Heiko Maurer ${ }^{*} \quad$ Katja Fiehler $^{* *} \quad$ Hermann Müller $^{*}$ \\ (*)Department of Psychology and Sport Science, Justus-Liebig-University, Giessen, Germany \\ (**)Department of Experimental and Biological Psychology, Philipps-University Marburg, \\ Germany \\ E-mail:mathias.reiser@sport.uni-giessen.de,heiko.maurer@sport.uni-giessen.de, \\ fiehler@staff.uni-marburg.de,hermann.mueller@sport.uni-giessen.de
}

\begin{abstract}
Whenever high target accuracy is a limiting factor of motor performance, as in goal-oriented throwing tasks, equifinal movement techniques can be used to reduce the influence of inherent biological variability on the task result. In throwing movements, for instance, these techniques are characterized by a specific spatio-temporal organization of the movement trajectory that allows several moments of release that lead to a target hit. Throwing experts (e.g. darts or boule players) with the experience of several ten thousand practice trials show equifinal movements, but also throwing novices can learn to use equifinality after at least 1000 trials of practice. Here, we present a study that aims to scrutinize how the acquisition of equifinal throwing movements can be improved and accelerated. Mechanical guidance was used as intervention method. Determination and mechanical implementation of an equifinal technique in a virtual goal-oriented throwing task as well as results of a four-day intervention experiment are presented. Results confirm that the approaches to compute an equifinal movement trajectory and to implement mechanical guidance are appropriate to support acquisition of error tolerant movement strategies.
\end{abstract}

\section{Introduction}

In motor tasks that require high target accuracy the inherent biological variability in movement execution should be especially disturbing. However, experts in goal-oriented tasks, like darts, basketball, or boules, are able to compensate deviations in execution variables by co-varying them. For instance, a release angle that is too small would be combined with a release velocity that can compensate the small angle with respect to the result or vice versa. As a consequence, this covariation improves target accuracy in these tasks $[1,2,3]$. The causal mechanism of these observations cannot be ascribed to feedback controlled corrections since in all these tasks the time window for release is too short to adjust one value depending on the other(s) (e.g. about $12 \mathrm{~ms}$ in darts). In fact, there is evidence that covariation arises from a defined spatio-temporal movement trajectory whose characteristic is a sequence of combinations of release parameters that hit the target [3]. In other words, this so called equifinal trajectory allows several release points throughout a longer time segment to hit the target and therewith reducing the negative influence of variability on the movement result. The moment of release can be freely varied and the target is nevertheless hit.

Reiser [2] could show that in darts, movement trajectories of the throwers became increasingly equifinal after 1000 practice trials without any special instruction. The present study aims to answer the question how the acquisition of an equifinal throwing movement can be improved and accelerated. The most direct intervention method would be the use of guidance, i.e. the equifinal throwing trajectory is conveyed to the performers by mechanical guidance from a servomotor controlled robot (IMOD). We tested whether the mechanical guidance had a positive effect on the acquisition of an equifinal throwing technique in a virtual throwing task and in consequence on the learning rate of the task.

\section{Methods}

\subsection{Experimental task}

The experimental task was a virtual throwing task (Skittles) whose origins lie in a British pub game. In this game, a ball is suspended from a string attached to

This is an Open Access article distributed under the terms of the Creative Commons Attribution-Noncommercial License 3.0, which permits unrestricted use, distribution, and reproduction in any noncommercial medium, provided the original work is properly cited. 
the tip of a vertical post. The player has to throw the ball around the post in order to knock down a target skittle on the other side. In the experimental version, participants saw the work space of the task in two dimensions from a bird's eye view on the projection surface from which they sat approximately $2 \mathrm{~m}$ away. They manipulated a lever with two degrees of freedom (shoulder and elbow) on the horizontal plane to gain velocity and released an electrical contact on the lever to release the virtual ball on the screen in front of them (Fig. 1).

In the guidance condition, the manipulation of the lever was executed by the IMOD; subjects only had to release the electrical contact to throw the ball. The lever was mechanically coupled with two servomotors that independently moved two slides in $\mathrm{x}$ and $\mathrm{y}$ direction on the horizontal plane (Fig. 1A). Therewith, the coordinates of the equifinal trajectory could be implemented in a passive movement of the subjects who had their arm rested on the lever, while instructed to actively follow the movement.

A

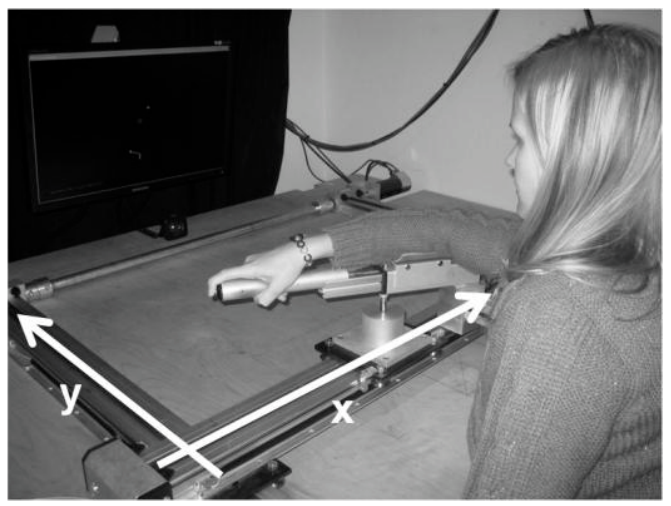

B

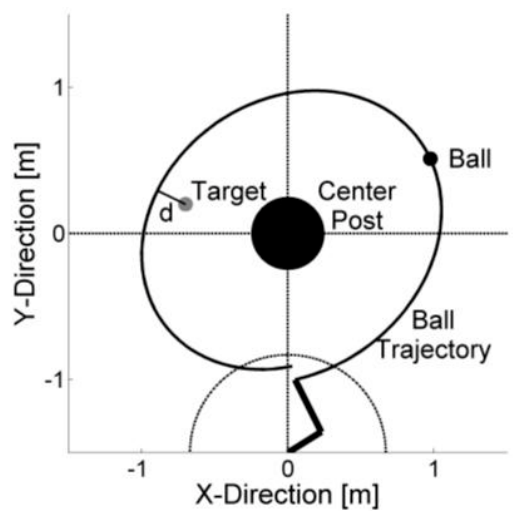

Fig. 1. Task setup (A) and task space projection (B)

Performance, as the minimal distance $\mathrm{d}$ between the trajectory of the ball and the center of the target (Fig. 1B), was determined by the execution variables position $(\mathrm{X}, \mathrm{Y})$ and velocity $\left(\mathrm{v}_{\mathrm{X}}, \mathrm{v}_{\mathrm{Y}}\right)$ at the moment of ball release. This distance was converted to a score from 0 to 100 points where 100 points were achieved with a distance equal to zero. The score decreased linearly with increasing distance to the target and reached zero for a distance equal or higher than $50 \mathrm{~cm}$ (including center post hits).

\subsection{Determination of the equifinal trajectory}

A numerical-iterative procedure is used to determine the equifinal trajectory along which the endeffector is guided.

Multiple equifinal trajectories exist for a throwing arm with two degrees of freedom. To specifically calculate one trajectory, an appropriate point of release within the XY-plane is defined first which serves as the starting point of the calculation. This release moment needs to fulfill the criteria to hit the target and to lead to a throwing movement that lies within the range of anatomical operability. From this starting point, the future hand position in the XY-plane after $1 \mathrm{~ms}$, when keeping the momentary velocity, is computed. The optimal velocity for this new position is determined by a minimal-jerk procedure, i.e. the velocity with minimal change in acceleration from the previous velocity is chosen. This procedure is conducted in a stepwise fashion for $40 \mathrm{~ms}$ before and after the moment of release, in steps of $1 \mathrm{~ms}$. As a result, we received an equifinal section of $80 \mathrm{~ms}$ duration, i.e. a sequence of hand positions in the XY-plane that in combination with the resulting velocities always lead to a target hit. Smooth start and end segments were added to the equifinal sequence to complete the movement.

\subsection{Participants and procedure}

28 female participants were randomly and equally assigned to either the group that received the guidance intervention or to the group without guidance. All subjects practiced Skittles for four days with 180 trials each. Nine test blocks of 40 trials at the beginning and at the end of each practice session and in an additional retention session one week after the last session were used to compare changes in performance and kinematic variables (equifinality of movements) due to practice. The intervention in the guidance group took place during the remaining 100 trials in between the two test blocks of a session. Concretely, after the first test block, 20 trials with guidance were followed by 20 trials without guidance (active condition), followed again by 20 trials with and 20 without guidance, followed by 10 trials with and 10 trials without guidance, closed by the second test block. The no- 
guidance group only practiced under the active condition for the whole time.

\subsection{Data analysis}

Throwing movements were analyzed at four different levels of description: (i) result-based level (distance to target, score), (ii) ball trajectories (equifinality measure), (iii) arm movement (shoulder and elbow angle), and (iv) release position (dpos).

Differences in performance improvement between both groups were analyzed with a 2 (group) $\times 9$ (block) ANOVA with repeated measures for the dependent variable score.

The use of equifinality in throwing movements was tested by determining the bundling of trajectories in the target area (Fig. 2). Concretely, for each trial we first calculated hypothetical trajectories for the time window $( \pm 40 \mathrm{~ms})$ in which equifinality should occur. The standard deviation (SD) of the distance values between these trajectories and the center of the target of all trials was then taken as a measure for the equifinality of that single throwing movement. For each test block the mean SD was then computed to compare the experimental groups.

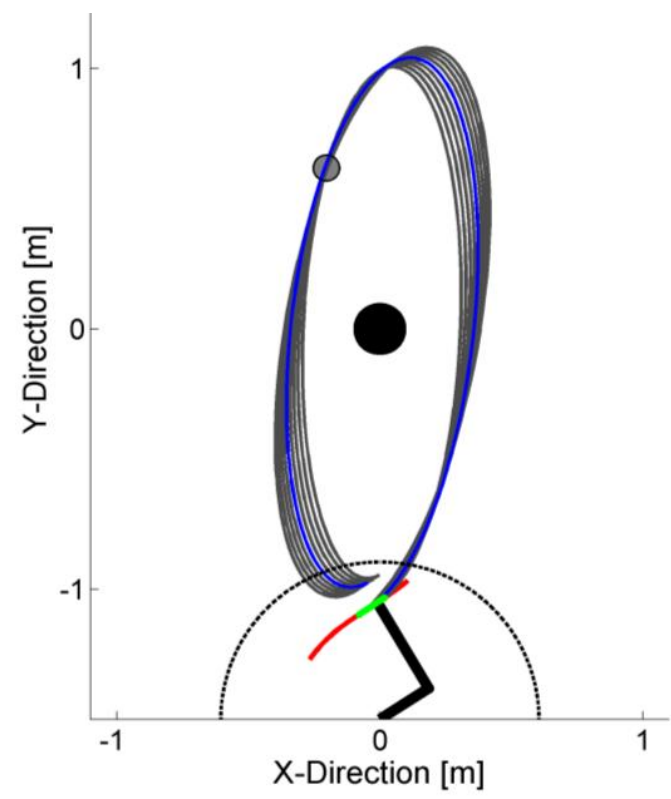

Fig. 2. Spatial path of the end-effector (red) including the equifinal segment (green) and the resulting real (blue) and hypothetical (grey) trajectories used to calculate the equifinality measure.

Arm movements were analyzed using angle-angle plots for elbow and shoulder angles. To quantify similarity between angle-angle characteristics in the guided and non-guided condition RMSE values were calculated (Fig. 4)

Due to the physical setup of this task, a release position right opposite to the target is optimal since it leads to hits irrespective of release velocity. We calculated the distance between this optimal release position and the mean actual release position of the subjects (dpos).

\section{Results}

Throwing performance significantly increased for both groups, but the test block $\times$ group interaction failed significance $(p=.28)$. However, the guidance group reached consistent performance scores faster as indicated by significant lower score variances for test block 2 and 3 ( $p=.020 ; p=.044)$.

Groups did not differ with respect to the equifinality measure $(p=.31)$.

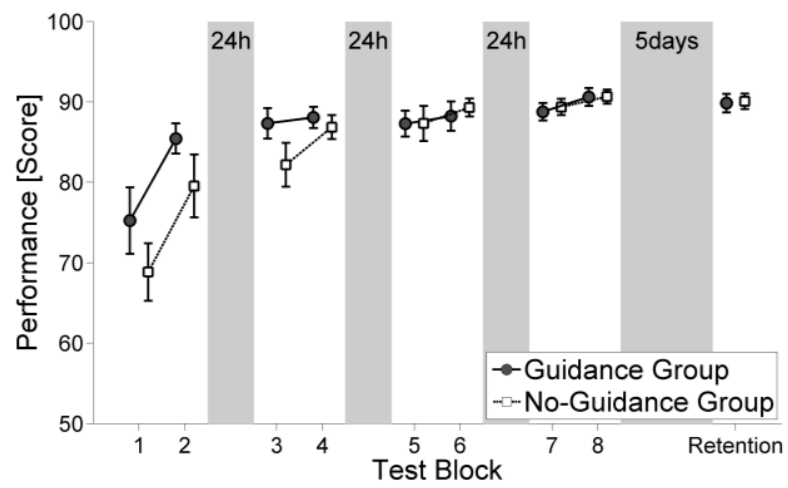

Fig. 3. Mean performance scores and standard errors.

Regarding the arm movement level of description, the guidance group did not approach the equifinal angular path (Fig. 4). RMSE-values of the guidance group did not differ from the no-guidance group.

However, already at the beginning of the intervention the guidance group realized release positions which were closer to the optimal release position. dpos guidance $<$ dpos no-guidance $(p=.002)$, i.e. leading to ball trajectories that were more tolerant to velocity fluctuations. 

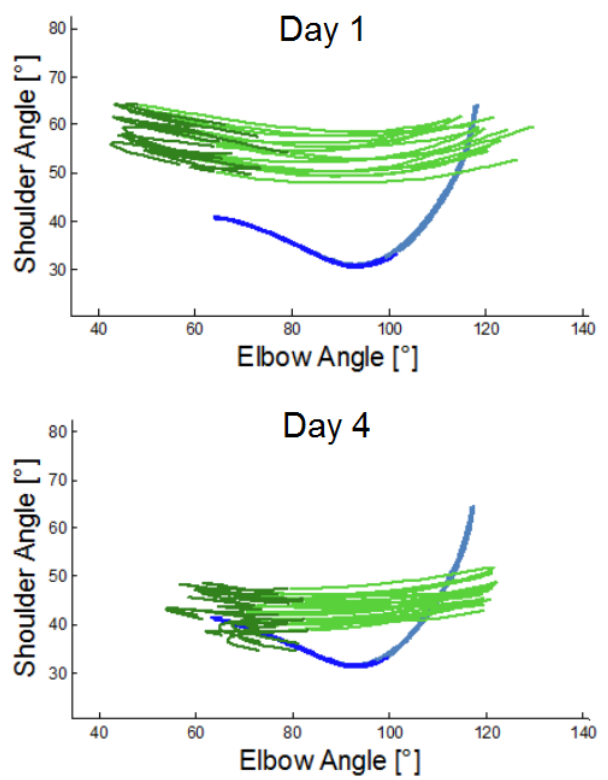

Fig. 4. Examples of angle-angle-plots of two series of throwing movements from a subject of the guidance group (green). The equifinal angular path, as predefined by the IMOD, is shaded in blue.

\section{Discussion}

The Skittles task can be optimally solved using an equifinal trajectory where the target is hit irrespective of release timing. We tried to enhance learning of this trajectory by providing strict physical guidance. Improvements became apparent only at the outcome level (score), but not in the arm movement itself. However, some spatial aspects of ball release were affected by the guidance intervention. In our case, physical guidance seems unsuitable for teaching predetermined movement trajectories, but rather conveys solution strategies at a spatial effect-related level of description (release).

Thus, guidance interventions that require subjects to more actively produce the movements (e.g. "haptic walls", [4]) might be more effective.

\section{References}

[1] K. Kudo, S. Tsutsui, T. Ishikura, T. Ito, and Y. Yamamoto. (2000). Compensatory coordination of release parameters in a throwing task. Journal of Motor Behavior, 32(4), pages. 337-345, 2000.

[2] M. Reiser. Zur Ergebniskonstanz von Bewegungstechniken beim Zielwurf, dissertationen.de, 2004.

[3] H. Müller, and E. Loosch. Functional variability and an equifinal path of movement during targeted throwing. Journal of Human Movement Studies, 36, pages. 103126, 1999.

[4] E.B. Brokaw, T. Murray, T. Nef, and P.S. Lum. Retraining of interjoint arm coordination after stroke using robot-assisted time-independent functional training. Journal of Rehabilitation Research \& Development, 48(4), pages. 299-316, 2011. 